\title{
Metabolic comorbidities and medical institution utilization among breast cancer survivors: a national population-based study
}

\author{
Jaesung $\mathrm{Heo}^{1}$, Mison Chun ${ }^{1}$, Young-Taek $\mathrm{Oh}^{1}$, O Kyu Noh ${ }^{1,2}$, and Logyoung $\mathrm{Kim}^{3}$
}

${ }^{1}$ Department of Radiation Oncology, ${ }^{2}$ Office of Biostatistics, Ajou University School of Medicine, Suwon; ${ }^{3}$ Health Insurance Review and Assessment Service, Seoul, Korea
Received: May 15, 2018

Revised : August 10, 2018

Accepted: September 6, 2018

\section{Correspondence to}

Mison Chun, M.D.

Department of Radiation Oncology, Ajou University School of

Medicine, 206 World cup-ro, Yeongtong-gu, Suwon 16499, Korea

Tel: $+82-31-219-5338$

Fax: +82-31-219-5894

E-mail: chunm@ajou.ac.kr
Background/Aims: We investigated metabolic comorbidity status and patterns of medical institution utilization among breast cancer survivors using medical claims data from the Health Insurance Review and Assessment Service (HIRA).

Methods: Using claims data obtained from the HIRA, we selected breast cancer survivors between 2010 and 2015. Descriptive statistics were calculated to determine the frequency of metabolic comorbidities, as well as to analyze patterns of medical institution utilization in accordance with disease status.

Results: A total of 89,953 breast cancer survivors were identified. Among these, 12,364 (13.7\%) had hypercholesterolemia, 20,754 (23.1\%) had hypertension (HTN), and $11,102(12.3 \%)$ had diabetes mellitus (DM). In particular, more than half of breast cancer survivors older than 60 years had HTN, and other diseases sharply increased beginning at age 50 years. For HTN, a total of 531,292 claims were submitted; more than $80 \%(n=473,737)$ were from primary medical institutions, whereas only $2.4 \%(n=12,551)$ were from tertiary medical institutions. The number of claims submitted for DM was 231,526; those from primary medical institutions accounted for $68.5 \%(\mathrm{n}=158,566)$, whereas claims from tertiary medical institutions accounted for $12.0 \%(n=27,693)$. In subgroup analyses, the utilization of secondary and tertiary medical institutions was higher among patients with severe diseases and those diagnosed following their breast cancer diagnosis.

Conclusions: More than $10 \%$ of breast cancer survivors were diagnosed with a metabolic comorbidity. Through analysis of medical institution utilization patterns, we ascertained that a communication system linking secondary and tertiary medical institutions with primary medical institutions is needed.

Keywords: Comorbidity; Breast neoplasms; Cancer survivors; Referral and consultation

\section{INTRODUCTION}

Breast cancer is the most commonly diagnosed cancer and the leading cause of cancer-associated death among women [1]. Due to early detection and improvements in treatment strategies, patients with breast cancer currently have improved prognoses and experience longer survival times than a decade ago [2]. In South Korea, the 5-year survival rate for breast cancer was > 90\% between 2009 and 2013 [3]. Moreover, in 2012, there were an estimated 1 million cancer survivors living in South Korea [4].

With respect to age, most cancer survivors are over 
60 years of age [5]. Therefore, cancer survivors are more likely to be diagnosed with cancer comorbidities [6]. As breast cancer is associated with obesity, breast cancer survivors experience metabolic comorbidities such as diabetes mellitus (DM), hypertension (HTN), and hypercholesterolemia (HC) [7-9]. Chemotherapy and hormonal therapy administered during cancer treatment can affect endocrine function and cause metabolic disease $[10,11]$. Diseases associated with breast cancer affect prognosis, and comorbidity-related mortality has become a focus of attention [12]. Moreover, metabolic diseases may reduce the quality of life of breast cancer survivors [13].

Chronic diseases in cancer survivors are not managed effectively [14]. In practice, HTN treatment compliance among cancer survivors was lower than that among healthy individuals [15]. Moreover, cancer survivors with DM were less likely to receive treatment than individuals without cancer, and were unable to achieve target glucose levels [16]. There are also differences in the complexity of treatment between diseases. Thus, appropriate management of metabolic comorbidities is important for cancer survivors. However, the status of metabolic comorbidities in breast cancer survivors has not been extensively investigated in South Korea.

In this study, we investigated the frequencies of metabolic comorbidities such as HTN, DM, and HC. In addition, we estimated patterns of medical institution utilization among breast cancer survivors with metabolic comorbidities using claims data from the Health Insurance Review and Assessment Service (HIRA).

\section{METHODS}

The Republic of Korea participates in a public medical insurance system known as the National Health Insurance (NHI) program. The HIRA reviews medical fees and services by collecting NHI claims data, including diagnosis, treatment, procedure, and medication information, for each patient. HIRA data are obtained from payment claims generated during patient visits to medical institutions. The HIRA database supplies information about patient sex, age, diagnosis, procedure, and prescriptions. Using HIRA claims data, we selected breast cancer survivors who were diagnosed with breast cancer and underwent surgery between January 2010 and July 2015. Numbers were used as surrogates for personal identifiers. The Institutional Review Board (IRB) of Ajou University Hospital approved this study (IRB No.: AJIRB-MED-EXP-15-538). The informed consent was waived by board.

The source population consisted of all patients who were diagnosed with a category C50 cancer according to the Korean Classification of Disease, sixth edition (KCD-6), a version of the International Classification of Disease 10 (ICD-10) modified for the Korean health care system. Breast cancer subgroups included nipple and areolar tumors (C500); tumors of the central portion of the breast ( $\mathrm{C}_{501}$ ); tumors of the upper-inner ( $\mathrm{C}_{502}$ ), lower-inner ( $\left.\mathrm{C}_{503}\right)$, upper-outer (C504), and lower-outer (C505) quadrants of the breast; tumors of the axillary tail of the breast (506); lesions overlapping the breast (C508); and breasts, unspecified (C509). We also used the following procedure codes: N7131 (simple mastectomy), N7133 (partial mastectomy), and N7135 (radical mastectomy). There was no lag time between the actual visit and claims data.

In order to identify breast cancer survivors with metabolic comorbidities, we used medical claims data from all patients, including those in the hospital, outpatient facilities, and emergency room, where in the principal diagnosis corresponded to one of three ICD-10 chronic disease categories: DM encompasses E1o (insulin-dependent DM), E11 (non-insulin-dependent DM), E12 (malnutrition-related DM), E13 (other specified DM), and E14 (unspecified DM), whereas HTN and HC are represented by I1o (hypertensive disease) and E78 (disorders of lipoprotein metabolism and other lipidemia), respectively. The following additional diseases were also analyzed to compare characteristics according to disease severity: ischemic heart disease (IHD), which is represented by I20 (angina pectoris), I21 (acute myocardial infarction), I22 (subsequent myocardial infarction), I23 (certain current complications following acute myocardial infarction), I24 (other acute IHDs), and I25 (chronic IHD), as well as cerebrovascular diseases (CVDs; I6).

Descriptive statistics were calculated, as appropriate, to estimate the frequency of each metabolic comorbidity. To analyze medical institution utilization patterns, we classified hospitals into one of three categories (primary medical institution, secondary medical institution, and 


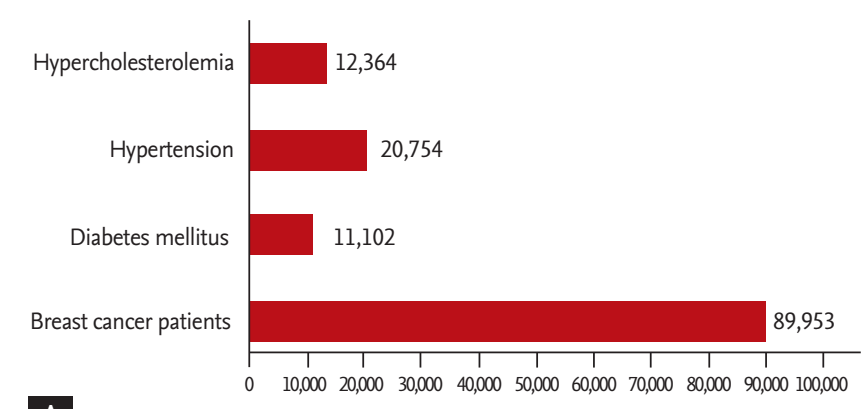

A

Figure 1. (A) Total number of breast cancer survivors diagnosed with each comorbidity. There were 12,364 patients with hypercholesterolemia (13.7\%), 20,754 with hypertension (23.1\%), and 11,102 with diabetes mellitus (12.3\%). (B) Comorbid disease distribution among breast cancer survivors; 32,983 breast cancer survivors (36.7\%) had at least one comorbidity, whereas 1,388 patients $(1.5 \%)$ had all three comorbidities.

Table 1. Breast cancer survivors with metabolic comorbidity characteristics

\begin{tabular}{ccccc}
\hline Variable & $\begin{array}{c}\text { Hypertension } \\
(\mathrm{n}=20,754)\end{array}$ & $\begin{array}{c}\text { Diabetes mellitus } \\
(\mathrm{n}=11,102)\end{array}$ & $\begin{array}{c}\text { Hypercholesterolemia } \\
(\mathrm{n}=12,364)\end{array}$ & $\begin{array}{c}\text { Breast cancer survivors } \\
(\mathrm{n}=89,953)\end{array}$ \\
\hline Age, yr & & & & $13(0.6)$ \\
$20-29$ & $6(0.3)$ & $12(0.6)$ & $357(2.4)$ & 14,971 \\
$30-39$ & $350(2.3)$ & $300(2.0)$ & $2,578(7.4)$ & 34,758 \\
$40-49$ & $3,935(11.3)$ & $2,017(5.8)$ & $5,272(23.0)$ & 22,960 \\
$50-59$ & $7,283(31.7)$ & $3,609(15.7)$ & $3,047(27.9)$ & 10,905 \\
$60-69$ & $5,971(54.8)$ & $3,285(30.1)$ & $1,009(26.6)$ & 3,799 \\
$70-79$ & $2,848(75.0)$ & $1,682(44.2)$ & $88(20.0)$ & 439 \\
$>80$ & $361(82.2)$ & $197(44.9)$ & & \\
\hline
\end{tabular}

Values are presented as number (\%).

tertiary medical institution) using institution-type identifiers corresponding to medical institution size. Primary medical institutions included clinics and public health centers, secondary medical institutions included hospitals and general hospitals, and tertiary medical institutions referred to advanced general hospitals. We evaluated patterns of medical institution utilization according to disease type, disease severity, and the time of diagnosis. All statistical analyses were performed with $\mathrm{R}$ 3.0.2 (R Foundation for Statistical Computing, Vienna, Austria).

\section{RESULTS}

In total, 89,953 breast cancer survivors who were diagnosed with breast cancer and underwent surgery between 2010 and 2015 were identified. The rates and types of comorbidities among breast cancer survivors are shown in Fig. 1. There were 12,364 patients with HC (13.7\%), 20,754 with HTN (23.1\%), and 11,102 with DM (12.3\%). HTN was the most frequently occurring comorbidity. In addition, 32,983 breast cancer survivors (36.7\%) had at least one comorbidity; 9,849 patients (10.9\%) had at least two comorbidities, with the combination of HTN and DM being the most common; and 1,388 patients $(1.5 \%)$ had all three comorbidities. Furthermore, IHD and CVD were identified at rates of $4.4 \%$ and $12.3 \%$, respectively.

Age group- and disease-specific frequency patterns are reported in Table 1 . The frequency of each metabolic comorbidity peaked among breast cancer survivors aged 50 to 59 years. However, the prevalence rate of metabolic comorbidities differed in accordance with age and dis- 
Table 2. Breast cancer survivors with IHD and CVD characteristics

\begin{tabular}{lccc}
\hline Variable & $\operatorname{IHD}(\mathrm{n}=3,949)$ & $\operatorname{CVD}(\mathrm{n}=11,102)$ & Breast cancer survivors $(\mathrm{n}=89,953)$ \\
\hline Age, $\mathrm{yr}$ & $2(0.9)$ & $5(0.2)$ & 2,121 \\
$20-29$ & $102(0.7)$ & $71(0.5)$ & 14,971 \\
$30-39$ & $583(1.7)$ & $558(1.6)$ & 34,758 \\
$40-49$ & $1,252(5.5)$ & $1,191(5.2)$ & 22,960 \\
$50-59$ & $1,223(11.2)$ & $1,247(11.4)$ & 10,905 \\
$60-69$ & $86(2.2)$ & $898(23.6)$ & 3,799 \\
$70-79$ & $3(0.7)$ & $151(34.4)$ & 439 \\
\hline 80 & & & \\
\hline
\end{tabular}

Values are presented as number (\%).

IHD, ischemic heart disease; CVD, cerebrovascular disease.

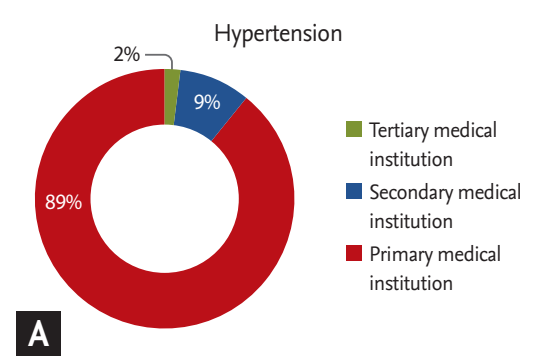

D
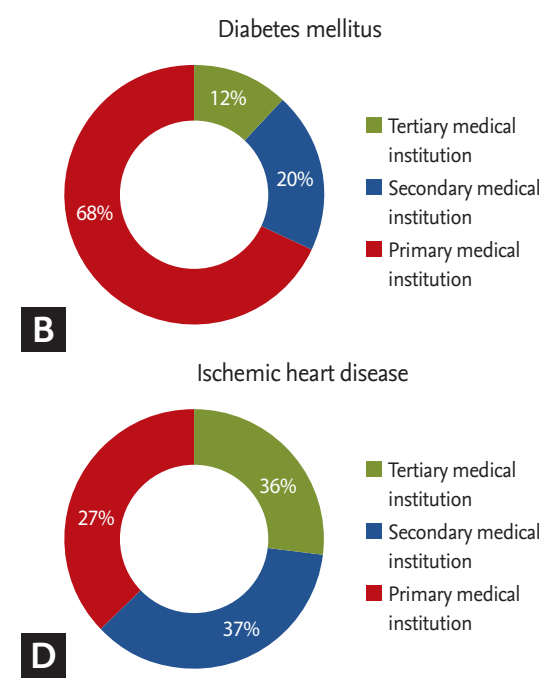

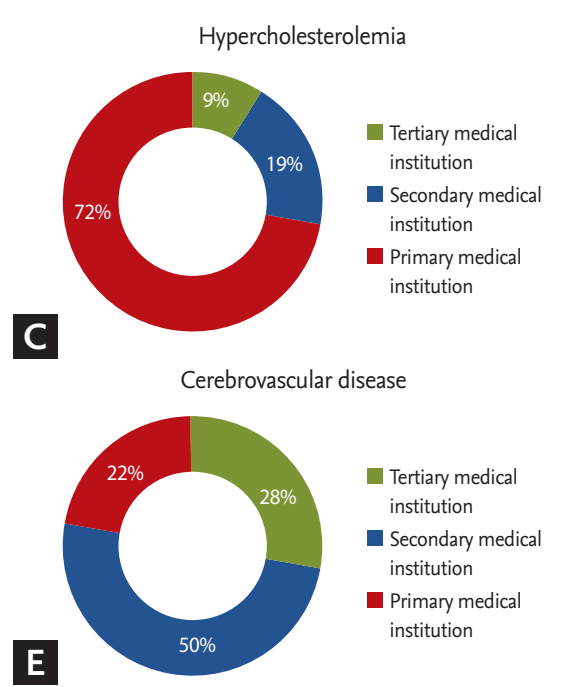

Figure 2. Patterns of medical institution utilization in accordance with disease type. (A) hypertension, (B) diabetes mellitus, (C) hypercholesterolemia, (D) ischemic heart disease, (E) cerebrovascular disease. The rates of secondary and tertiary med-ical institution utilization were high among patients with severe diseases such as ischemic heart disease and cardiovascular disease.

ease group; metabolic disease prevalence increased with age. In particular, HT'N was found in more than half of breast cancer survivors aged 60 years or older. In addition, the prevalence of DM increased rapidly beginning at age 60 years, whereas that of $\mathrm{HC}$ rose beginning at age 50 years. The proportion of CVD was relatively low, but a rate of $20 \%$ was observed for those older than 70 years (Table 2).

Differences in medical institution utilization were observed among patients with HTN (Fig. 2). In total, there were 531,292 claims submitted for HTN. Over 80\% $(473,737)$ of claims for HT'N treatment were submitted by primary medical institutions, whereas those from tertiary medical institutions accounted for only $2.4 \%(12,551)$. In addition, claims from primary medical institutions accounted for 158,565 (68.5\%) of DM claims, whereas those from secondary and tertiary medical institutions accounted for 72,960 (31.5\%). The utilization rate of secondary and tertiary medical institutions was also relatively high (27.7\%) among breast cancer survivors with HC. The utilization rate of secondary and tertiary medical institutions was also high among patients with CVD (78\%) and IHD (73\%). With respect to the time of diagnosis of metabolic diseases, a greater proportion of 

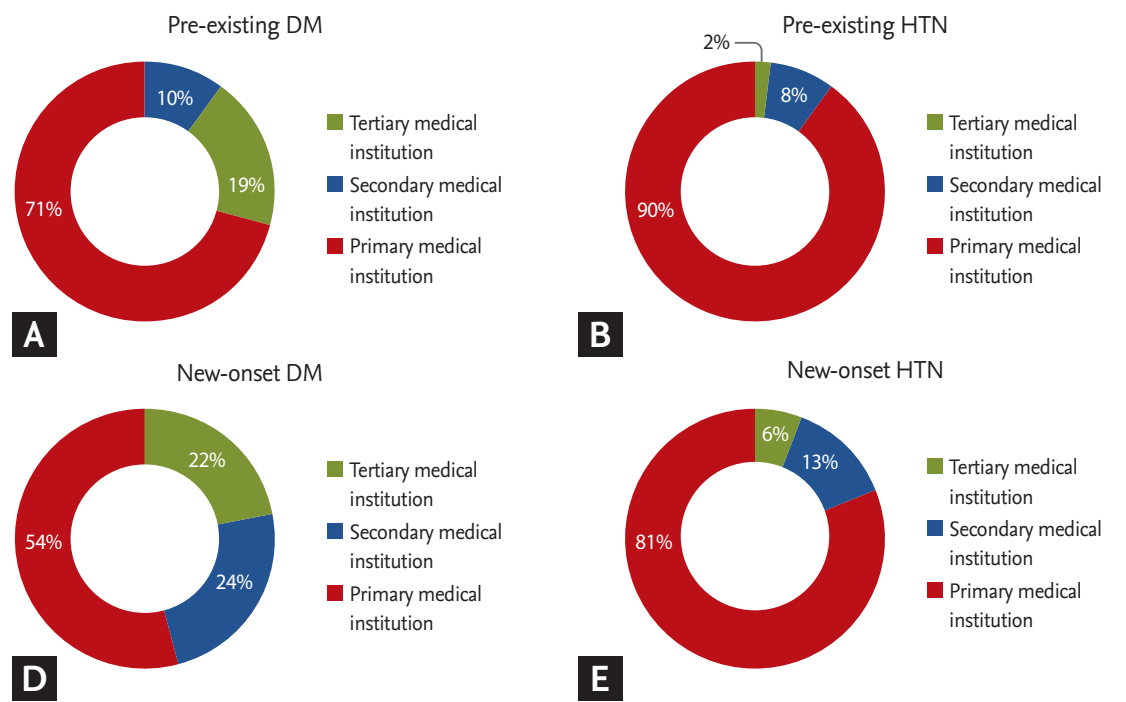

C
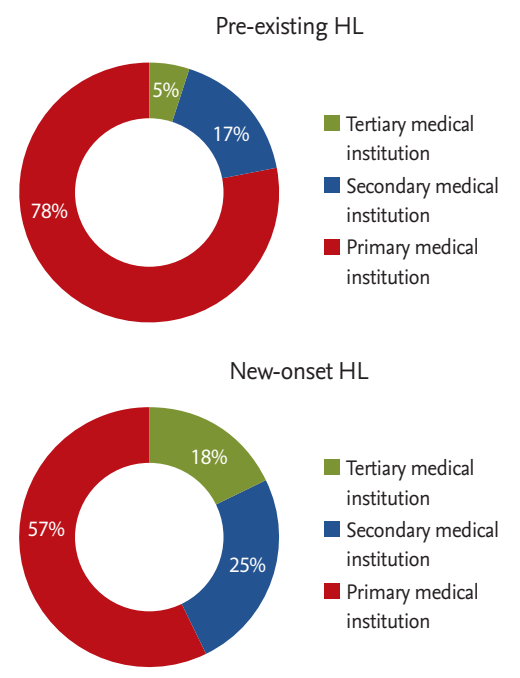

Figure 3. Patterns of medical institution utilization based on time of diagnosis. When diagnosed with a comorbidity after a breast cancer diagnosis, rates of secondary and tertiary medical institution use were high. (A) Pre-exisiting diabetes mellitus (DM), (B) pre-existing hypertension (HTN), (C) pre-existing hypercholesterolemia (HC), (D) new-onset DM, (E) new-onset HTN, and (F) new-onset HC.

patients utilized secondary and tertiary medical institutions after their breast cancer diagnosis (Fig. 3).

\section{DISCUSSION}

Breast cancer survivors are more likely to experience metabolic comorbidities such as DM, HTN, and HC [7-9]. In our study, we determined that $36.7 \%(32,983)$ of breast cancer survivors in South Korea were diagnosed with at least one comorbidity, and the age range with the peak incidence was 50 to 59 years (Fig. 1). In particular, the metabolic disease was shown to be $10 \%$ to $20 \%$ among all breast cancer survivors, but was 30\% higher among those aged 50 to 60 years (Table 1). It is therefore necessary to intensively evaluate metabolic disorders among women in this age range who are undergoing breast cancer treatment.

A previous study on the management of HTN and DM in the entire population found that patients with these conditions was $14 \%$ and $5 \%$, respectively, between July 2014 and June $2015[17,18]$. In our study, the patients among breast cancer survivors with HTN and DM was significantly high (23\% and $12 \%$, respectively). Moreover, the prevalence rate of $17 \%$ was found among
United States breast cancer survivors with DM [19]. In addition, $9.2 \%$ of leukemia survivors present with metabolic diseases [20]. Breast cancer diagnoses occur at relatively younger ages among Asian women than among their Western counterparts, and breast cancer survivors have good prognoses [21]. Therefore, it is important to properly manage metabolic comorbidities and maintain long-term treatment compliance. We focused on patterns of medical institution utilization with respect to patients' metabolic comorbidities.

Breast cancer survivors utilized primary care in different ways depending on the comorbidity with which they were diagnosed. Over $60 \%$ of breast cancer survivors visited primary medical institutions for management of their metabolic comorbidity. Survivors with HTN had the highest frequency of treatment in primary medical institutions (Fig. 2). On the other hand, patients with DM were more likely to receive treatment in secondary or tertiary medical institutions. Differences in facility use could be due to the relative complexities of medication regimens. In a previous study in which medication regimen complexity (MRC) was estimated with scores based on dosage form, frequency, and the need for additional directions, the MRC score for DM was higher than that for HTN [22]. In addition to requir- 
ing glycemic control, patients with DM need to manage complications such as DM-related foot problems [23]. In addition, $59.4 \%$ of patients with DM, but only $41.8 \%$ of patients with HTN, presented with another comorbidity (Fig. 1). Thus, breast cancer survivors with DM may be more likely to visit higher-level institutions such as secondary or tertiary medical centers.

There were no differences in patterns of medical institution use according to the occurrence of complications in the diabetic group (Supplementary Fig. 1). This result is due to the high proportion of patients with diabetes who used secondary or tertiary medical institutions. On the other hand, we observed differences in medical institution use according to disease severity (Fig. 3). In case of cardiovascular and CVDs, the proportion of patients who used secondary or tertiary medical institutions was greater than 70\%. This result is thought to be an example of disease severity affecting differences in utilization of medical institutions. In addition, the difference in utilization patterns according to the time of metabolic disease diagnosis indicates that secondary and tertiary medical institutions are highly used when patients are diagnosed with chronic diseases after their breast cancer diagnosis. This result may be attributed to the fact that metabolic diseases are revealed on various tests performed during the breast cancer diagnostic process, and that treatment is continuously administered thereafter. Therefore, it is important to develop different approaches for managing metabolic diseases in breast cancer survivors that depend on both disease severity and the time of diagnosis.

We determined that greater than two-thirds of breast cancer survivors with a comorbidity used a primary medical institution for treatment (Fig. 2). However, the referral system in which primary care doctors connect patients to a large hospital, or vice versa, is insufficient in South Korea. Moreover, medical institution-based cancer survivorship clinics can only be operated by tertiary medical centers located in metropolitan areas [6]. However, continuous follow-up in the hospital where primary treatments such as surgery, chemotherapy, and radiotherapy were performed is necessary. Therefore, cancer survivors need an efficient healthcare referral system to link the surgical hospital to local private clinics and public health centers for further disease management.

In European countries, hospitals and local clinics have clearly delineated functions and complementary relationships. Primary care physicians act as gatekeepers to access to hospital specialists. However, in South Korea, the relationship between hospitals and private clinics is competitive, which is the result of unclear roles among healthcare institutions. As a result, significant medical expenses are wasted on unnecessary healthcare services [24]. Therefore, an effort must be made to distinguish between the roles of specialists in hospitals and primary physicians in private clinics.

Furthermore, a shared-care model to facilitate communication between specialists and primary physicians is needed [25]. It is recommended that medical institutions share the medical histories of patients undergoing treatment with local primary care providers. However, primary care providers in South Korea lack the knowledge and experience to manage cancer survivors. Moreover, cancer survivors who were treated by primary physicians in local clinics were less likely to undergo secondary cancer screening and surveillance [26]. Therefore, educational programs and guidelines based on the healthcare environment in South Korea, rather than that in Western countries, are necessary for providing relevant information to primary physicians responsible for management of cancer survivors.

A systematic, government-run cancer survivor care plan is also necessary. In developed countries, the government has tried to improve patient flow from hospitals to local medical institutions after treatment. For example, in Australia, regional cancer centers were established to address the gap in medical accessibility between rural and metropolitan areas [27]. Similarly, in South Korea, 12 regional cancer centers were established between 2004 and 2012 to provide clinical care for cancer survivors. Therefore, a community, regional cancer center-based survivorship care plan needs to be developed to overcome cancer care inequality among cancer survivors.

This study should be interpreted in light of its limitations. First, we identified comorbidity cases based on HIRA claims data, which only includes claims codes and demographic information. Clinical data such as hormone receptor status, cancer staging, and imaging and laboratory test results were not available. Future studies combining HIRA claims data with clinical datasets such as NHI service data are necessary. Second, we could only 
use diagnostic codes to evaluate the presence of a metabolic disease according to time sequence. Therefore, future studies should examine whether treatment-related factors, such as receipt of radiotherapy and chemotherapy, are also associated with metabolic disease occurrence. Third, data pertaining to recurrence or metastasis among breast cancer survivors were not available in the claims data. Finally, it was impossible to compare hospital utilization patterns between breast cancer survivors with metabolic comorbidities and non-breast cancer patients with metabolic diseases.

In conclusion, with an aging population and an increasing number of patients diagnosed with cancer, managing metabolic comorbidities is important for improving survivors' quality of life. Through an analysis of medical institution utilization patterns, we found that a referral system linking hospitals and private clinics is necessary. Additionally, we should consider a regional cancer center-based, shared-care model to promote communication between specialists in hospitals and primary physicians in local areas.

\section{KEY MESSAGE}

1. It is important to properly manage the metabolic comorbidity and maintain treatment compliance over a long period.

2. Through analysis of medical institution utilization patterns, we found that a referral system linking hospitals and private clinics was necessary.

3. We could consider a shared-care model to promote communication between specialists in hospitals and primary physicians in local area.

\section{Conflict of interest}

No potential conflict of interest relevant to this article was reported.

\section{REFERENCES}

1. Siegel RL, Miller KD, Jemal A. Cancer statistics, 2016. CA Cancer J Clin 2016;66:7-30.

2. Arrington AK, Goldstein L, Kruper L, Vito C, Yim J, Chen
SL. Life expectancy after curative-intent treatment of breast cancer: impact on long-term follow-up care. Am Surg 2014;80:604-609.

3. Oh CM, Won YJ, Jung KW, et al. Cancer statistics in Korea: incidence, mortality, survival, and prevalence in 2013. Cancer Res Treat 2016;48:436-450.

4. Jung KW, Won YJ, Kong HJ, Oh CM, Lee DH, Lee JS. Cancer statistics in Korea: incidence, mortality, survival, and prevalence in 2011. Cancer Res Treat 2014;46:109-123.

5. DeSantis CE, Lin CC, Mariotto AB, et al. Cancer treatment and survivorship statistics, 2014. CA Cancer J Clin 2014;64:252-271.

6. Lee JE, Shin DW, Lee H, et al. One-year experience managing a cancer survivorship clinic using a shared-care model for gastric cancer survivors in Korea. J Korean Med Sci 2016;31:859-865.

7. Peairs KS, Barone BB, Snyder CF, et al. Diabetes mellitus and breast cancer outcomes: a systematic review and meta-analysis. J Clin Oncol 2011;29:40-46.

8. Goon PK, Messerli FH, Lip GY. Hypertension and breast cancer: an association revisited? J Hum Hypertens 2006;20:722-724.

9. Nelson ER, Wardell SE, Jasper JS, et al. 27-Hydroxycholesterol links hypercholesterolemia and breast cancer pathophysiology. Science 2013;342:1094-1098.

10. Hickish T, Astras G, Thomas P, et al. Glucose intolerance during adjuvant chemotherapy for breast cancer. J Natl Cancer Inst 2009;101:537.

11. Redig AJ, Munshi HG. Care of the cancer survivor: metabolic syndrome after hormone-modifying therapy. Am J Med 2010;123:87.

12. Chen WW, Shao YY, Shau WY, et al. The impact of diabetes mellitus on prognosis of early breast cancer in Asia. Oncologist 2012;17:485-491.

13. Tang Z, Wang J, Zhang H, et al. Associations between diabetes and quality of life among breast cancer survivors. PLoS One 2016;11:e0157791.

14. Earle CC, Neville BA. Under use of necessary care among cancer survivors. Cancer 2004;101:1712-1719.

15. Shin DW, Park JH, Park JH, et al. Antihypertensive medication adherence in cancer survivors and its affecting factors: results of a Korean population-based study. Support Care Cancer 2010;19:211-220.

16. Shin JY, Shim HY, Jun JK. Comparison of diabetes management status between cancer survivors and the general population: results from a Korean population-based sur- 
vey. PLoS One 2014;9:e110412.

17. Health Insurance Review and Assessment Service. Evaluation of Appropriateness of Medical Care Benefits for Diabetes Mellitus. Wonju (KR): HIRA, 2016.

18. Health Insurance Review and Assessment Service. Evaluation of Appropriateness of Medical Care Benefits for Hypertension. Wonju (KR): HIRA, 2016.

19. Shao S, Gill AA, Zahm SH, et al. Diabetes and overall survival among breast cancer patients in the U.S. military health system. Cancer Epidemiol Biomarkers Prev 2018;27:50-57.

20. Oudin C, Simeoni MC, Sirvent N, et al. Prevalence and risk factors of the metabolic syndrome in adult survivors of childhood leukemia. Blood 2011;117:4442-4448.

21. Youlden DR, Cramb SM, Yip CH, Baade PD. Incidence and mortality of female breast cancer in the Asia-Pacific region. Cancer Biol Med 2014;11:101-115.

22. Rettig SM, Wood Y, Hirsch JD. Medication regimen complexity in patients with uncontrolled hypertension and/ or diabetes. J Am Pharm Assoc (2003) 2013;53:427-431.
23. Armstrong DG, Cohen K, Courric S, Bharara M, Marston W. Diabetic foot ulcers and vascular insufficiency: our population has changed, but our methods have not. J Diabetes Sci Technol 2011;5:1591-1595.

24. Lee JY, Jo MW, Yoo WS, Kim HJ, Eun SJ. Evidence of a broken healthcare delivery system in korea: unnecessary hospital outpatient utilization among patients with a single chronic disease without complications. J Korean Med Sci 2014;29:1590-1596.

25. Oeffinger KC, McCabe MS. Models for delivering survivorship care. J Clin Oncol 2006;24:5117-5124.

26. Shin DW, Kim Y, Baek YJ, Mo HN, Choi JY, Cho J. Oncologists experience with second primary cancer screening: current practices and barriers and potential solutions. Asian Pac J Cancer Prev 2012;13:671-676.

27. Walsh J, Harrison JD, Young JM, Butow PN, Solomon MJ, Masya L. What are the current barriers to effective cancer care coordination? A qualitative study. BMC Health Serv Res 2010;10:132. 

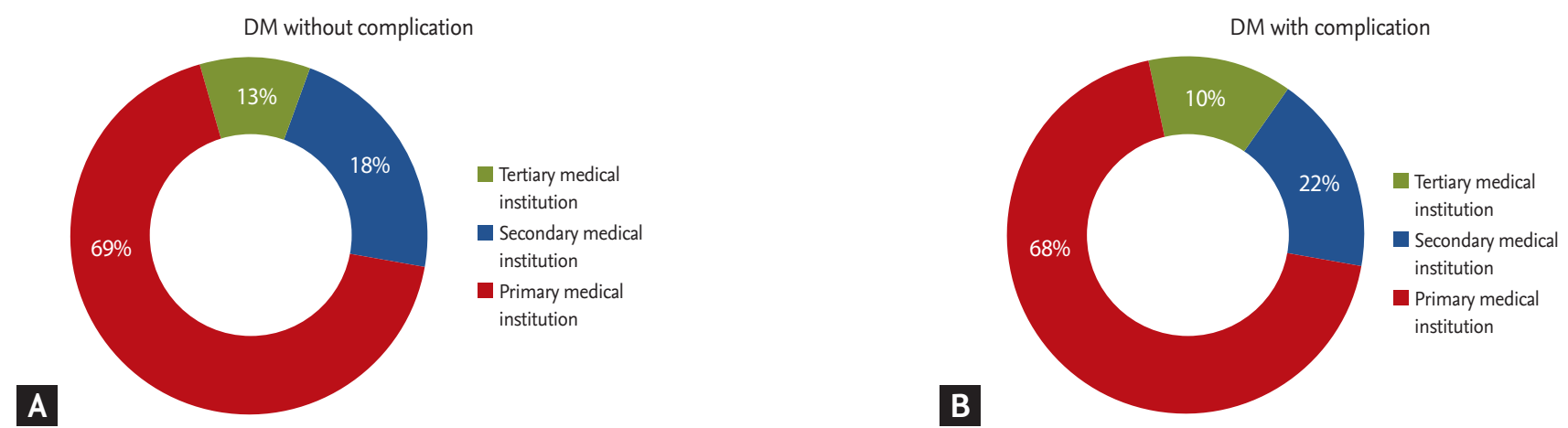

Supplementary Figure 1. Patterns of medical institution utilization based on complication among patients with diabetes mellitus (DM). There were no differences in hospital utilization behaviors according to diabetic complication. (A) DM without complication and (B) DM with complication. 